\title{
Size and reflection effects in priming: A test of transfer-appropriate processing
}

\author{
KAVITHA SRINIVAS \\ Boston College, Chestnut Hill, Massachusetts
}

\begin{abstract}
Prior research has suggested that priming on perceptual implicit tests is insensitive to changes in stimulus size and reflection. The present experiments were performed to investigate whether size and reflection effects can be obtained in priming under conditions that encourage the processing of this information at study and at test, as predicted by transfer-appropriate processing. The results indicate that priming was affected by a change in the physical size of an object when study and test tasks required a judgment about the real size of pictorial objects (e.g., deciding whether a zebra presented small or large on the screen was larger or smaller than a typical chair), and when the test task required the identification of fragmented pictures. However, a change in left-right orientation had no effect on priming when study and test tasks required a judgment about the left-right orientation of familiar objects, or when the test task involved the identification of fragmented pictures. This difference between size and reflection effects is discussed in terms of the differential importance of size and reflection information in shape identification.
\end{abstract}

When we perceive objects in everyday situations, we must represent specific perceptual information (e.g., their size, left-right orientation, etc.) about these objects to aid later identification and recognition. Recent research suggests that the perceptual information utilized in later object identification (i.e., naming or judgments that require the structural analysis of an object) differs from the information utilized in episodic recognition (i.e., in the recollection of a specific encounter with an object). The typical paradigm used to demonstrate this dissociation involves contrasting memory performance on two types of memory tests. In implicit memory tests, studied objects are re-presented in a test phase along with nonstudied objects, and participants are asked to name the objects as quickly as possible (see, e.g., Biederman \& E. E. Cooper, 1991a, 1991b, 1992) or are asked to judge the three-dimensional plausibility of the objects (e.g., L. A. Cooper, Schacter, Ballesteros, \& Moore, 1992). The measure of implicit memory is the degree of facilitation (or priming) in naming or object decisions for studied objects relative to nonstudied objects. In explicit memory tests, participants are presented the same set of studied and nonstudied objects, but are asked if they can recollect whether they studied a given object earlier. The interesting finding is that performance on implicit mem-

Part of this research was presented at the 1993 meeting of the Midwestern Psychological Association in Chicago. I would like to thank Teresa Blaxton for her helpful comments on this article, and Suparna Rajaram, Henry L. Roediger III, and John Schwoebel for comments on an earlier version of this paper. I would also like to thank Patty Hoyos and Caroline Fitzgerald for assistance in testing participants in the experiments reported here. Correspondence should be addressed to K. Srinivas, Department of Psychology, Boston College, Chestnut Hill, MA 02167-3807 (e-mail: srinivas@ $($ hermes.bc.edu). ory tests is not affected by changes in the size or leftright orientation of studied objects, whereas performance on explicit memory tests is adversely affected by studyto-test changes in size and left-right orientation (see, e.g., Biederman \& E. E. Cooper, 1991a, 1992; L. A. Cooper et al., 1992; Jolicoeur, 1987; Milliken \& Jolicoeur, 1992).

One interpretation of this finding is that implicit memory tests tap structural object representations that specify the shape of objects in terms of their parts and the spatial relations between the different parts. Because changes in size or in left-right orientation do not affect the description of the object in terms of its parts or its spatial relations, these changes are assumed to have a minimal effect on implicit memory performance (Biederman \& E. E. Cooper, 1991a, 1992; L. A. Cooper et al., 1992). In contrast, explicit memory tests are hypothesized to tap episodic representations of objects that include distinctive spatial, temporal, and contextual information about objects (L. A. Cooper et al., 1992). Because changes in size or in left-right orientation alter the distinctive spatial attributes of objects, they are assumed to have an adverse effect on recognition memory.

Further, some theorists have postulated that structural and episodic representations of objects are simultaneously formed in two separate memory systems whenever an object is perceived. By these accounts, implicit memory tests that require the perceptual analysis of objects (such as naming, identification of three-dimensional plausibility, naming of fragmented objects, etc.) employ the perceptual representation system (PRS) or the procedural memory system dedicated to the perception of objects, whereas explicit memory tests tap an episodic or declarative memory system that is involved in the conscious recollection of objects, words, and events (e.g., Squire, 
1992; Tulving \& Schacter, 1990). The PRS for visually presented objects is assumed to be based in the inferior temporal cortex (IT), because data from the neuroscience literature suggest that it is the terminal region responsible for higher level object identification (Schacter, 1992). Indeed, data from cell-recording and lesion studies indicate that the IT is responsible for object identification across changes in stimulus size, reflection, and location (see Miyashita, 1993; and Plaut \& Farah, 1990, for reviews), and this is consistent with the results obtained with priming. The episodic system is assumed to be dependent on the hippocampus and is thought to include input from several other cortical systems, such as the shape-based system in the IT (or the "what" system) and the system involved in visuospatial processing (or the "where" system) (Biederman \& E. E. Cooper, 1992; Squire, 1992; Ungerleider \& Mishkin, 1982).

Apart from the neural analysis of dissociations between explicit and implicit memory tests discussed above, processing differences between explicit and implicit tests also account for some dissociations. This processing analysis of explicit-implicit dissociations is provided by the transfer-appropriate processing framework. The basic tenet of this approach is that performance on memory tests benefits most when encoding operations overlap maximally with retrieval demands of a particular test (Kolers \& Roediger, 1984; Morris, Bransford, \& Franks, 1977; Roediger, 1990). By this account, for most explicit tests and conceptual implicit tests that emphasize the semantic relationship between the studied and tested word (e.g., when participants are asked to produce exemplars to category names such as animal after studying an exemplar such as elephant in another context), the overlap in encoding and retrieval processes must occur at a semantic level in order to facilitate performance (Blaxton, 1989; Jacoby, 1983; Roediger, Weldon, \& Challis, 1989; Srinivas \& Roediger, 1990). In contrast, for perceptual implicit tests that emphasize a perceptual relationship between studied and tested words (e.g., when participants are asked to name fragmented words such as $\mathrm{e}_{-} \mathrm{e} \mathrm{p}_{-} \mathrm{n}_{-}$after studying elephant), the overlap in encoding and retrieval processes must occur at a perceptual level.

The transfer-appropriate processing framework provides a reasonable explanation of some dissociations with explicit and implicit memory tests in the verbal domain (see Roediger \& McDermott, 1993, for a recent review). Thus, for example, explicit and conceptual implicit tests are affected by meaningful elaboration at study (Blaxton, 1989; Hamann, 1990; Rappold \& Hashtroudi, 1991; Srinivas \& Roediger, 1990), whereas perceptual implicit tests are affected primarily by changes in the perceptual aspects of a studied word, such as its modality (e.g., Blaxton, 1989). However, the transferappropriate processing approach cannot readily account for dissociations between explicit and implicit nonverbal tests due to changes in size or reflection. Because these are perceptual changes, and because the implicit memory tests in question are perceptual as well, one would expect that the match in size or reflection between study and test should affect perceptual implicit tests such as naming and object decision rather than conceptual explicit tests such as recognition memory. However, one could still argue within this framework that size and reflection information have no effect on naming or on impossible/ possible decisions, because they are irrelevant to task performance on these tests. Of course, this is a post hoc explanation of size and reflection effects, but this does not mean that the framework cannot be used to successfully predict experimental outcomes. In particular, the principle has been useful in prior research in demonstrating that dissociations are possible within perceptual implicit tests that tap the same memory system when the task demands of the perceptual tests differ. A selective review of these studies is provided below.

The best example of dissociations between two perceptual implicit tests is the effect of a change in domain (pictures or words) between study and test. Priming on a picture fragment completion task is enhanced when pictures are presented at study relative to when words are presented at study, but the reverse is true for word fragment completion tasks (see, e.g., Srinivas \& Roediger, 1990; Weldon \& Roediger, 1987). According to the transferappropriate processing framework, the dissociation occurs because perceptual implicit tests such as fragment completion are sensitive to the perceptual match between study and test stimuli, and this match is better in the case of studied words on a word fragment completion test and studied pictures on a picture fragment completion test. Further, the account predicts that priming on these perceptual tests should be sensitive to type of stimuli presented at study and test. For instance, priming should be greater on word and picture fragment completion when the same fragmented word or picture appears both at study and at test than when an intact or a different fragmented version is presented at study. This finding has now been obtained in both word fragment completion (Gardiner, Dawson, \& Sutton, 1989) and picture fragment completion (Srinivas, 1993; but see Snodgrass \& Feenan, 1990, and Biederman \& E. E. Cooper, 1991b, for exceptions).

Similarly, it could be argued from the transferappropriate processing principle that priming on a picture fragment completion test should be enhanced when fragmented pictures rather than intact pictures are presented at study, but intact picture naming performance should show the reverse pattern. This prediction has been confirmed in recent experiments by Snodgrass and Hirshman (1994) on two explicit and two implicit memory tasks. In these experiments, participants studied objects that were fragmented at different levels (ranging from Level 1, which was the most fragmented version, to Level 7, which was the almost complete version). On the two explicit tests, the participants were presented with fragmented or intact versions of the object for episodic recognition. On the two implicit tests, the participants were presented with the same fragmented or intact versions for naming. The results indicated better perfor- 
mance when study and test conditions matched (intactintact or fragmented-fragmented) than when they did not match (intact-fragmented or fragmented-intact), regardless of the explicit/implicit nature of the tests. These findings suggest that for both explicit and implicit memory tests, the critical determinant of memory test performance is the match in encoding and retrieval operations; that is, the processing of fragmented figures at test is facilitated most by the processing of similar fragmented figures at study, whereas the processing of intact pictures at test is facilitated most by the processing of complete figures at study.

The crucial question that these studies raise is whether the transfer-appropriate processing framework can be used to make specific predictions about size and leftright orientation effects on perceptual implicit tests. On the basis of this principle, one would predict that changes in size or left-right orientation should affect implicit memory performance if task demands at encoding and retrieval require the processing of this information. Converging evidence was sought in the present experiments by exploring this issue across several implicit and explicit memory tasks. Experiments 1 and 2 explored the effects of encoding and retrieval demands on the representation of size in priming tasks, and these findings were contrasted with a recognition memory test in Experiment 3. Experiments 4 and 5 explored the effects of encoding and retrieval demands on the representation of left-right orientation in priming, and these results were contrasted with data from recognition memory tests in Experiments 6 and 7. Because the manipulation of encoding and retrieval demands in these experiments was achieved primarily through study and test tasks, these tasks are described briefly below.

In Experiment 1, priming was assessed on a size judgment task that was designed to encourage the encoding of size at study and at test. The task required participants to decide whether an object presented on the screen was typically larger or smaller than a "standard" chair. The physical size of the object was also manipulated so that the object appeared large or small on the computer screen. Previous studies (Davidoff \& Ostergaard, 1988; Paivio, 1975) with variants of this task have reported a Stroop-like interference when the physical size of the object on the screen was incompatible with its size in memory (e.g., a zebra presented small on the screen or a telephone presented large on the screen). These findings imply that the task requires the encoding of object size, even when it is irrelevant to task performance. Therefore, it was predicted that if participants performed the size judgment task at study and at test, size information about objects would be important both at encoding and at retrieval and would therefore be included in perceptual object representations. In Experiment 2, encoding of size was encouraged by the use of the size judgment task, but priming was assessed at test on another perceptual implicit task, picture fragment naming. Although the fragment naming task does not explicitly require the retrieval of size information about objects, it was hypothesized that size compatibility between study and test would have an effect on the task because size alterations of the fragments can affect the gaps in the contour for a particular object. Further, because fragment naming seems sensitive to the contours presented at study and test so that performance is actually better for degraded versions of studied objects rather than complete versions (Snodgrass \& Hirshman, 1994; Srinivas, 1993), it was predicted that the lack of overlap in contour between study and test due to a change in size would affect priming. Experiment 3 was an attempt to replicate previous findings obtained with an explicit recognition memory test (Biederman \& E. E. Cooper, 1992; L. A. Cooper et al., 1992; Jolicoeur, 1987; Milliken \& Jolicoeur, 1992), but with the same size judgment encoding task as that used in Experiments 1 and 2 at study.

In Experiment 4, the use of left-right information was encouraged at study and test by asking participants to decide whether familiar objects faced left or right on the computer screen. When used at encoding with novel objects, the left-right task has been reported to promote the creation of three-dimensional descriptions of the structure of objects relative to other tasks that require elaborative processing (Schacter, Cooper, \& Delaney, 1990). The interesting question is whether the use of the task at encoding and retrieval would result in effects of left-right orientation on priming. Experiments 5-7 also required the encoding of left-right orientation information at study, but the test tasks were either fragment completion (Experiment 5) or recognition (Experiments 6 and 7), for reasons already outlined.

\section{EXPERIMENT 1}

\section{Method}

Participants. Forty-eight Boston College undergraduates participated in this experiment in partial fulfillment of a course requirement.

Materials. Forty-eight digitized line drawings of objects were selected from the Snodgrass and Vanderwart (1980) norms. Large versions of the line drawings could be contained in a circle with a diameter that subtended a visual angle of $17.22^{\circ}$. Small versions were created by reducing the line drawings by $50 \%$ on both $x$ and $y$ dimensions. Small versions could be contained in a circle with a diameter that subtended a visual angle of $8.8^{\circ}$.

Design. A 3 (study condition: small, large, or nonstudied) $\times 2$ (test condition: small or large) $\times 2$ (response: larger than a chair, smaller than a chair) within-subjects design was used. Of the 48 line drawings, 24 represented objects that were larger than chairs, and the other 24 represented objects that were smaller than chairs in real life. The 48 items were then divided into six blocks of 8 items each (4 representing objects larger than chairs, and 4 smaller than chairs). Each block was rotated across the six studytest conditions to ensure that each item appeared equally often in each condition across participants. Study and test items were presented in a different random order for each participant. Both latency data and accuracy data were the dependent measures on this task.

Procedure. The participants were tested in small groups, on three Dell computers. In the study phase, they were instructed that 
they would see a set of line drawings of objects on the computer screen that would vary in size. They were asked to make a judgment about whether the object represented by the line drawing was larger than a prototypical chair, or smaller than a chair. They were instructed to base this size judgment on their knowledge about the real size of the objects, and they were told to ignore the size variations of the objects on the computer screen. The participants were also asked to make this judgment as quickly and as accurately as possible, by pressing a green (left) mouse key if the object was larger than a chair, and a red (right) mouse key if it was smaller than a chair. If they failed to respond by pressing the mouse key within $5 \mathrm{sec}$, the next stimulus was presented. The participants were also instructed to use their preferred hand in making the response. A C program was used in this (and all subsequent experiments) to control stimulus presentation and to record the latency and accuracy data. In the study phase, 10 practice items and 32 target pictures were presented to each participant.

At the end of the study phase, participants were engaged in an unrelated distractor task for $5 \mathrm{~min}$. The instructions given in the test phase were identical to the instructions given at study, except that participants were also informed that the experimenter was interested in observing the effects of practice on the size judgment task. No mention was made of the relationship between study and test items. Ten practice items were presented before the presentation of the 48 target items.

\section{Results and Discussion}

The following conventions will be adopted in reporting all the experiments in this paper. All the results reported as being reliable exceeded the .05 level of confidence, unless specified otherwise. The results of the analyses of variance (ANOVAs) and the $t$ tests are reported on the basis of subject $\left(F_{1}\right)$ or item $\left(F_{2}\right)$ variability. Further, because in most of the experiments the primary dependent measure is latency, the latency data are discussed first, followed by the accuracy data. Tables $1 \mathrm{~A}-1 \mathrm{~B}$ show the latency and accuracy data on the size judgment task as a function of the different conditions at study and at test.

The results indicate that participants encoded size at study, despite being told to ignore the changes in the size of the object on the computer screen. Specifically, the participants showed a Stroop-like interference when objects larger than chairs in real life were presented in a small size on the screen (91-msec effect), and conversely, when objects smaller than chairs were presented in a large size on the screen (98-msec effect). Repeated measures ANOVAs confirmed that this interaction between the response (larger or smaller than a chair) and the studied size (large or small) was reliable $\left[F_{1}(1,47)=\right.$ $\left.15.83, M S_{\mathrm{e}}=28,359 ; F_{2}(1,46)=15.57, M S_{\mathrm{e}}=17,176\right]$. Similarly, in the accuracy data, only the interaction between the response and studied size was reliable $\left[F_{1}(1,47)\right.$ $\left.=6.77, M S_{\mathrm{e}}=.005 ; F_{2}(1,46)=4.24, M S_{\mathrm{e}}=.004\right]$. These results replicate previous findings reported by Davidoff and Ostergaard (1988) and Paivio (1975). No other effects from the study phase approached significance.

The results on the size judgment task at test are presented separately in Table 1B for the two types of responses (larger or smaller than a chair). As shown in the table, the latency data indicate, once again, an interaction between the type of response (larger or smaller than a chair) and the physical size of the stimulus on the screen at test (large or small), suggesting a Stroop-like interference at test when the physical size of the object on the screen did not match the response $\left[F_{1}(1,47)=\right.$ $\left.15.22, M S_{\mathrm{e}}=23,982 ; F_{2}(1,46)=27.25, M S_{\mathrm{e}}=10,137\right]$, and suggesting that size information was utilized at test as well. Because the nature of the response (larger or smaller than a chair) was not relevant for the priming

Table 1A

Accuracy (PC, Proportion Correct) and Latency (RT, in Milliseconds) for the Size Judgment Task at Study in Experiment 1

\begin{tabular}{lccccc} 
& \multicolumn{4}{c}{ Response } \\
\cline { 2 - 3 } $\begin{array}{c}\text { Study } \\
\text { Condition }\end{array}$ & "Larger than a chair" & & "Smaller than a chair" \\
\cline { 2 - 3 } \cline { 5 - 6 } & RT & PC & & RT & PC \\
Small & 1,165 & .91 & 1,022 & .97 \\
Large & 1,074 & .94 & 1,120 & .94 \\
Interference & 91 & .03 & 98 & .03 \\
\hline
\end{tabular}

Table $1 \mathrm{~B}$

Accuracy (PC, Proportion Correct) and Latency (RT, in Milliseconds) for the Size Judgment Task at Test in Experiment 1

\begin{tabular}{|c|c|c|c|c|c|c|c|c|c|c|c|c|}
\hline \multirow{4}{*}{$\begin{array}{c}\text { Study } \\
\text { Condition }\end{array}$} & \multicolumn{8}{|c|}{ Response } & \multirow{2}{*}{\multicolumn{4}{|c|}{$\begin{array}{c}\text { Combined } \\
\text { Across Response }\end{array}$}} \\
\hline & \multicolumn{4}{|c|}{ "Larger than a chair" } & \multicolumn{4}{|c|}{ "Smaller than a chair" } & & & & \\
\hline & \multicolumn{2}{|c|}{ Small } & \multicolumn{2}{|c|}{ Large } & \multicolumn{2}{|c|}{ Small } & \multicolumn{2}{|c|}{ Large } & \multicolumn{2}{|c|}{ Small } & \multicolumn{2}{|c|}{ Large } \\
\hline & RT & $\mathrm{PC}$ & RT & PC & RT & $\mathrm{PC}$ & RT & $\overline{\mathrm{PC}}$ & RT & $\mathrm{PC}$ & $\overline{\mathrm{RT}}$ & $\overline{\mathrm{PC}}$ \\
\hline Small & 87 & .94 & 867 & .97 & 835 & .99 & 871 & .96 & 85 & .97 & 869 & .97 \\
\hline Large & 937 & .94 & 783 & .98 & 854 & .99 & 824 & .96 & 896 & .97 & 804 & .97 \\
\hline Nonstudied & 1,074 & .93 & 962 & .97 & 908 & .99 & 935 & .94 & 992 & .96 & 948 & .96 \\
\hline
\end{tabular}

Note-The column headings "Small" and "Large" refer to the physical size of the stimulus on the screen at test. The row headings "Small" and "Large" refer to the physical size of the stimulus on the screen at study 
analysis, the data were collapsed across the two types of responses. Instead, the data were analyzed for the size match and size mismatch across study and test.

As expected, priming was obtained for studied items; facilitation relative to the nonstudied small or nonstudied large conditions was observed in the small-small (139 $\mathrm{msec})$, large-large (144 $\mathrm{msec})$, large--small (96 msec), and small-large ( $79 \mathrm{msec})$ study conditions. These priming effects were larger for conditions that maintained size between study and test (small-small and large-large) relative to conditions that involved a size change between study and test (large-small and smalllarge). Repeated measures ANOVAs confirmed these observations. The priming effect (difference between studied and nonstudied items) was reliable in both the subject and the item analyses $\left[F_{1}(1,47)=25.64, M S_{\mathrm{e}}=\right.$ 12,$\left.258 ; F_{2}(1,47)=16.15, M S_{\mathrm{e}}=20,817\right]$. Priming scores were computed and subjected to a separate analysis to explore the effects of maintaining or changing size between study and test. There was a reliable main effect of study-test compatibility in size $\left[F_{1}(1,47)=8.49\right.$, $\left.M S_{\mathrm{e}}=15,429 ; F_{2}(1,47)=7.78, M S_{\mathrm{e}}=13,395\right]$. This effect did not interact with the size of the stimulus at test $(F \mathrm{~s}<1)$, suggesting that the advantage of maintaining size was obtained on both large and small items at test. Analysis of the accuracy data revealed no statistically reliable effects, primarily because performance was at ceiling.

Two major findings emerged in this experiment: (1) The size judgment task appeared to require the encoding of size information even when participants were explicitly instructed to ignore size information while performing the task, and (2) as expected, priming was adversely affected by size changes between study and test when the study and test tasks required the processing of size information. Experiment 2 was an attempt to replicate this effect of size alteration on a picture fragment naming task.

\section{EXPERIMENT 2}

\section{Method}

Participants. Sixty-six Boston College undergraduates participated in this experiment in partial fulfillment of a course requirement.

Materials. Only one of the line drawings used in Experiment 1 was replaced because an appropriate picture fragment could not be constructed for the object ( $c l o u d$ ). Corresponding picture fragments for the 48 line drawings selected in Experiment 1 were constructed by randomly deleting $8 \times 8$ pixel blocks in the image until approximately $80 \%$ of the contour had been deleted from the image. Contour deletion was done first on the large images by a $C$ program. Subsequently, these fragmented versions were reduced by $50 \%$ on both the $x$ and $y$ dimensions. A sample of these images is provided in Figure 1.

Design. A 3 (study condition: small, large, or nonstudied) $\times 2$ (test condition: small or large) within-subjects design was used. As in Experiment 1, 24 of the 48 line drawings represented objects that were larger than chairs, and the other 24 represented objects that were smaller than chairs. Other design details were identical
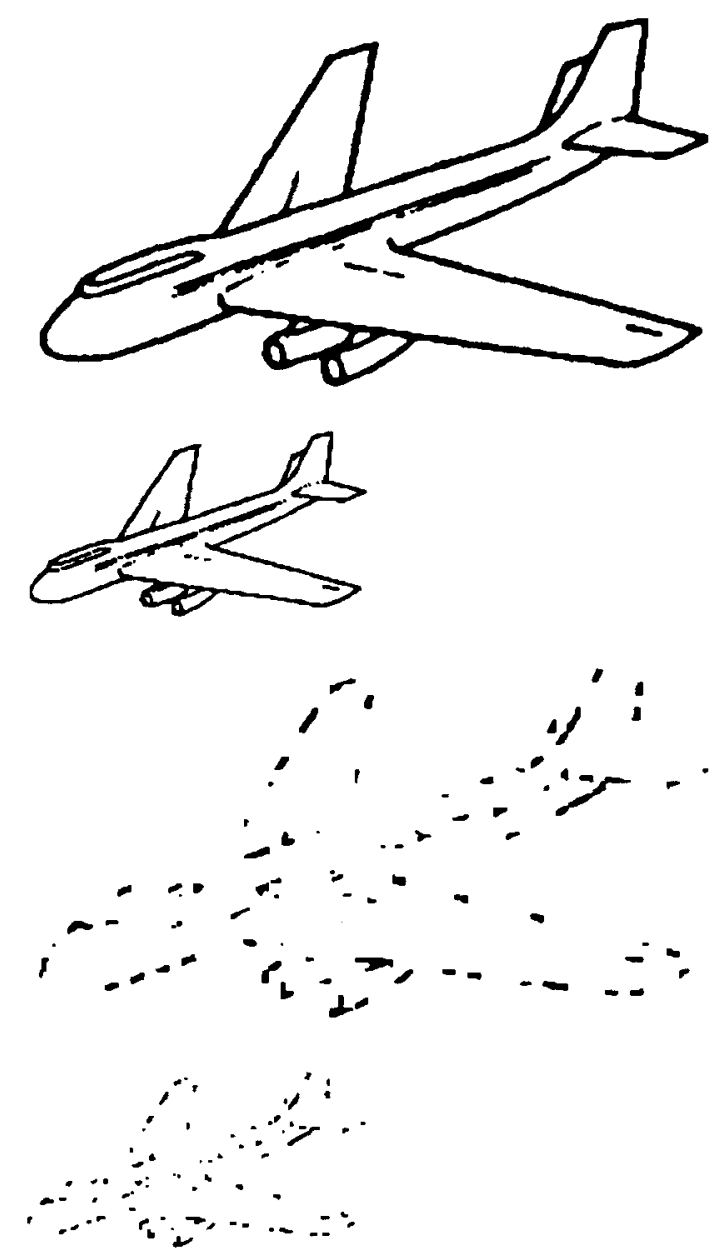

Figure 1. Examples of intact and fragmented versions of objects shown in the two sizes.

to those in Experiment 1. The task at test was picture fragment naming.

Procedure. The participants were tested in small groups, with three Dell computers. The procedures used in the study phase were identical to the procedures in Experiment 1. At the end of the study phase, the participants were engaged in an unrelated distractor task for $5 \mathrm{~min}$. They were then instructed that they would see a new set of line drawings of objects that were incomplete or fragmented. They were asked to press the green (left) mouse key as soon as they could identify the object, and to type the correct name for the object. The object was replaced by a prompt to enter the name of the object as soon as the participants pressed the mouse key. The next trial was initiated by another keypress. If the subject failed to respond within a period of $5 \mathrm{sec}$, he/she was presented with the next item. The participants were encouraged to guess the identity of the object and to respond with the first name that came to mind. Ten practice items were presented before the presentation of the 48 target items.

\section{Results and Discussion}

Tables $2 \mathrm{~A}-2 \mathrm{~B}$ provide a summary of the results of Experiment 2 , for both the size judgment task at study and the picture fragment completion task at test. 
Table 2A

Accuracy (PC, Proportion Correct) and Latency (RT, in Milliseconds) in the Size Judgment Task at Study in Experiment 2

\begin{tabular}{|c|c|c|c|c|}
\hline \multirow{3}{*}{$\begin{array}{c}\text { Study } \\
\text { Condition }\end{array}$} & \multicolumn{4}{|c|}{ Response } \\
\hline & \multicolumn{2}{|c|}{ "Larger than a chair" } & \multicolumn{2}{|c|}{ "Smaller than a chair" } \\
\hline & RT & $\mathrm{PC}$ & RT & $\mathrm{PC}$ \\
\hline Small & 1,151 & .91 & 1,130 & .93 \\
\hline Large & 1,075 & .95 & 1,140 & .91 \\
\hline Interference & 76 & .04 & 10 & .02 \\
\hline
\end{tabular}

Table 2B

Accuracy (PC, Proportion Correct) and Latency (RT, in Milliseconds) in the Picture Fragment Naming Task at Test in Experiment 2

\begin{tabular}{lccccc}
\hline & \multicolumn{4}{c}{ Test Size } \\
\cline { 2 - 3 } \multicolumn{1}{c}{ Study } & \multicolumn{2}{c}{ Small } & & \multicolumn{2}{c}{ Large } \\
\cline { 2 - 3 } \cline { 5 - 6 } Condition & RT & PC & & RT & PC \\
\hline Small & 1,562 & .73 & & 1,419 & .83 \\
Large & 1,754 & .76 & & 1,430 & .88 \\
Nonstudied & 2,021 & .49 & & 1,900 & .62 \\
\hline
\end{tabular}

Note-Test size ("Small," "Large") refers to the physical size of the stimulus on the screen at test. The row headings "Small" and "Large" refer to the physical size of the stimulus on the screen at study.

The results at study indicated once again that participants processed size information while performing the size judgment task, even though size was irrelevant to task performance. Thus, the participants were slower at classifying objects larger than chairs when the size of the object was small on the screen as opposed to when it was large on the screen (a 76-msec effect in latency, 4\% difference in accuracy). However, this pattern was not obtained with objects that were smaller than chairs (there was only a 10 -msec advantage in latency, and a $2 \%$ advantage to classifying objects that were small relative to those presented large). Repeated measures ANOVAs on the latency data indicated that the interaction between response type (larger or smaller than a chair) and size of the object at study (small or large) was reliable or approached significance $\left[F_{1}(1,64)=4.83, M S_{\mathrm{e}}=32,882\right.$; $\left.F_{2}(1,46)=3.27, M S_{\mathrm{e}}=17,735 ; p<.07\right]$. A similar interaction was observed in the accuracy data $\left[F_{1}(1,65)=\right.$ $\left.2.94, M S_{\mathrm{e}}=.02, p<.09 ; F_{2}(1,47)=6.19, M S_{\mathrm{e}}=.02\right]$.

The results of the picture fragment naming task at test indicated a large benefit in latency and accuracy respectively for the small-small (459 $\mathrm{msec}, .24)$, large-large (470 msec, .26), large-small ( $267 \mathrm{msec}, .27$ ), small-large (481 msec, .21) studied conditions. Reliable facilitation occurred in the studied conditions relative to the nonstudied conditions in the latency $\left[F_{1}(1,63)=79.22\right.$, $\left.M S_{\mathrm{e}}=77,959 ; F_{2}(1,37)=40.56, M S_{\mathrm{e}}=199,754\right]$ and accuracy $\left[F_{1}(1,65)=169.30, M S_{\mathrm{e}}=.01 ; F_{2}(1,47)=\right.$ $\left.72.82, M S_{\mathrm{e}}=0.02\right]$ data. To explore the effect of maintaining or changing size between study and test, priming scores in the four conditions were again subjected to a separate analysis. The data indicated an effect of studytest compatibility in size in the latency $\left[F_{1}(1,63)=4.64\right.$,
$M S_{\mathrm{e}}=122,777 ; F_{2}(1,37)=3.09, M S_{\mathrm{e}}=156,348 ; p<$ $.08]$, but not in the accuracy data $(F \mathrm{~S}<1)$. However, the interaction between study-test compatibility and the size of fragments at test was reliable in the subject analysis of the latency data $\left[F_{1}(1,63)=5.41, M S_{\mathrm{e}}=93,353\right.$; $\left.F_{2}(1,37)=2.28, M S_{\mathrm{e}}=110,645 ; p<.13\right]$ and in the accuracy data $\left[F_{1}(1,65)=5.65, M S_{\mathrm{e}}=.02 ; F_{2}(1,47)=\right.$ $\left.7.74, M S_{\mathrm{e}}=0.01\right]$. Individual $t$ tests revealed larger priming effects in latency for small fragments presented in the same size at study and test than for those that were changed in size $\left[t_{1}(65)=3.03, t_{2}(47)=2.07\right]$. A similar advantage for fragments maintained in size occured for large fragments, but only in the accuracy data $\left[t_{1}(65)=\right.$ $2.09, t_{2}(47)=2.29$ ]. No other effects reached significance. Overall, the data suggest an effect of changing size on picture fragment naming, on either the accuracy or the latency measures.

The pattern of results from Experiments 1 and 2 suggests that size information is included in the representations that mediate priming, when size needs to be processed at study or at test. In other words, size effects occurred in the two experiments primarily because the processing of size information was encouraged both at study and at test. These results support a transferappropriate processing account of memory test performance, and attests to the flexibility with which objects are represented in the perceptual representation systems (Roediger \& Srinivas, 1993).

Experiment 3 was designed to replicate the effects of size on recognition memory reported in prior studies (Biederman \& E. E. Cooper, 1992; L. A. Cooper et al., 1992; Jolicoeur, 1987; Milliken \& Jolicoeur, 1992) with the procedures and materials used in Experiments 1 and 2.

\section{EXPERIMENT 3}

\section{Method}

Participants. Thirty Boston College undergraduates participated in this experiment in partial fulfillment of a course requirement.

Materials and Design. The materials and design of this experiment were identical to those in Experiment 1.

Procedure. The participants were tested in small groups, with three Dell computers. In the study phase, the participants were asked to discriminate objects that were larger than chairs from objects smaller than chairs. The study procedures were identical to those in Experiments 1 and 2. At the end of the study phase, the participants were engaged in an unrelated distractor task for $5 \mathrm{~min}$. Following the distractor tasks, they were instructed to discriminate studied objects from nonstudied objects as quickly and as accurately as possible, and they were instructed to ignore any variations in size in making their judgments. The participants were asked to press the green (left) mouse key to indicate studied objects, and the red (right) mouse key to indicate nonstudied objects. Ten filler items were presented along with the 48 target items at test.

\section{Results and Discussion}

Tables $3 \mathrm{~A}-3 \mathrm{~B}$ present the accuracy and latency data for the size judgment task at study and the recognition memory task at test. 
Table 3A

Accuracy (PC, Proportion Correct) and Latency (RT, in Milliseconds) in the Size Judgment Task at Study in Experiment 3

\begin{tabular}{lcccc}
\hline & \multicolumn{4}{c}{ Response } \\
\cline { 2 - 3 } \multicolumn{1}{c}{ Study } & "Larger than a chair" & "Smaller than a chair" \\
\cline { 3 - 6 } Condition & RT & PC & RT & PC \\
\hline Small & 1,029 & .91 & 1,036 & .96 \\
Large & 962 & .97 & 970 & .93 \\
Interference & 67 & .06 & -66 & .03 \\
\hline
\end{tabular}

Table 3B

Accuracy (PC, Proportion Correct) and Latency (RT, in Milliseconds) in the Recognition Memory Task at Test in Experiment 3

\begin{tabular}{lccccc}
\hline & \multicolumn{4}{c}{ Test Size } \\
\cline { 2 - 3 } \multicolumn{1}{c}{$\begin{array}{c}\text { Study } \\
\text { Condition }\end{array}$} & \multicolumn{2}{c}{ Small } & & \multicolumn{2}{c}{ Large } \\
\cline { 2 - 3 } \cline { 5 - 6 } & RT & PC & & RT & PC \\
\hline Small & 889 & .97 & & 928 & .92 \\
Large & 926 & .93 & & 848 & .96 \\
Nonstudied & 1,044 & .86 & & 1,038 & .86
\end{tabular}

Note-Test size ("Small," "Large") refers to the physical size of the stimulus on the screen at test. The row headings "Small" and "Large" refer to the physical size of the stimulus on the screen at study.

The results of the size judgment task at study are surprising, because they do not completely replicate the pattern found in Experiments 1 and 2. Although there was interference in the processing of objects that were larger than chairs when the objects were presented small on the screen, a similar interference was not obtained for objects that were smaller than chairs. For the small objects, a speed-accuracy tradeoff was observed such that in the accuracy data, the size congruency effect was in the expected direction, but this was reversed in the latency data. These observations were confirmed by repeated measures ANOVAs; the interaction between response (larger or smaller than a chair) and the physical size of the object at study (large or small) was not reliable by participants or by items in the latency data $\left(F_{1}, F_{2}<1\right)$, but was reliable in the accuracy data $\left[F_{1}(1,29)=7.57\right.$, $\left.M S_{\mathrm{e}}=.01 ; F_{2}(1,46)=6.39, M S_{\mathrm{e}}=.01\right]$. Further, the main effect of studied size was reliable in the latency data $\left[F_{1}(1,29)=6.90, M S_{\mathrm{e}}=20,224 ; F_{2}(1,46)=8.94\right.$, $\left.M S_{\mathrm{e}}=10,605\right]$, indicating that small objects were processed more slowly than large objects. However, this effect was not reliable in the accuracy data.

Together, the results for the size judgment task at study do indicate a Stroop-like interference effect for objects larger than chairs such that the physical size of the object on the computer screen interfered with the judgment about real size. However, this effect was not obtained with objects smaller than chairs, although it was obtained in Experiment 1 and to a smaller extent in Experiment 2. There is no apparent reason for this discrepancy, because the participants were treated identically in the study phase and were presented with the same materials in all three experiments. Despite the discrepancy in the data, it is clear that the participants were encoding size information at study in the present experiment, because large processing differences were found between small and large views in the latency data, and a Strooplike interference effect was obtained with one of the responses. The next issue of interest was the effect of encoding size information on the recognition memory task.

As expected, the results at test indicated an effect of maintaining size between study and test relative to changing size between study and test. Thus, better performance was obtained for the large-large condition than for the small-large condition $(80 \mathrm{msec}$ and .04 difference in latency and accuracy, respectively), and better performance was obtained for the small-small condition than for the large-small condition $(37 \mathrm{msec}$, and .04 difference in latency and accuracy, respectively). The effect of maintaining size between study and test was reliable in both the latency data $\left[F_{1}(1,29)=4.58, M S_{\mathrm{e}}=\right.$ 22,$\left.577 ; F_{2}(1,47)=6.71, M S_{\mathrm{e}}=38,825\right]$ and the accuracy data $\left[F_{1}(1,29)=6.85, M S_{\mathrm{e}}=.01 ; F_{2}(1,47)=6.61\right.$, $\left.M S_{\mathrm{e}}=.01\right]$, suggesting once again that recognition memory is affected by changes in size even when participants are explicitly asked to ignore size changes in making their judgments about the study status of a particular object.

Clearly, Experiments 1-3 provide evidence that size changes affect performance on both explicit and implicit memory tests when the processing of size is encouraged at study and at test. Experiments 4-7 examined whether similar results would be obtained with another perceptual attribute (left-right orientation). Experiment 4 was designed to explore the effects of encoding left-right orientation information at study on a task that also required the processing of left-right orientation at test.

\section{EXPERIMENT 4}

\section{Method}

Participants. Forty Boston College undergraduates participated in this experiment in partial fulfillment of a course requirement.

Materials. Eighty Snodgrass and Vanderwart (1980) line drawings were selected for this experiment. The objects had a clear left-right orientation.

Design. A 2 (study status: studied or nonstudied) $\times 2$ (orientation at study: left or right) $\times 2$ (orientation at test: left or right) within-subjects design was used. The 80 items were then divided into eight blocks of 10 items each. Each block was rotated across the eight study-test conditions to ensure that each item appeared equally often in each condition across participants. Study and test items were presented in a different random order for each subject. Both the latency data and the accuracy data were the dependent measures on this task.

Procedure. The participants were tested in small groups, with three Dell computers. In the study phase, the participants were asked to discriminate objects that faced left from objects that faced right, as quickly and as accurately as possible. The participants responded by pressing the green (left) mouse key for objects that were oriented left, and the red (right) mouse key for objects that were oriented right. The participants used their preferred hands to perform the task. At the end of the study phase, the participants were engaged in an unrelated distractor task for 5 min. 
Table 4

Accuracy (PC, Proportion Correct) and Latency (RT, in Milliseconds) in the Left-Right Judgment Task at Test in Experiment 4

\begin{tabular}{llllll}
\hline & \multicolumn{4}{c}{ Test Orientation } \\
\cline { 2 - 4 } \multicolumn{1}{c}{ Study } & \multicolumn{2}{c}{ Left } & & \multicolumn{2}{c}{ Right } \\
\cline { 2 - 4 } Orientation & RT & PC & & RT & PC \\
\hline Left & 559 & .98 & & 565 & .98 \\
Right & 555 & .98 & & 565 & .97 \\
Nonstudied & 594 & .97 & & 582 & .97 \\
\hline
\end{tabular}

Following the distractor task, the participants were instructed to perform the left-right judgment again to 80 target items ( 40 studied and 40 nonstudied).

\section{Results and Discussion}

Table 4 presents the latency and accuracy data on the left-right judgment task at test. (The results at study indicated no differences between objects that were oriented left and those that were oriented right. This result is not surprising, because objects were assigned to the left or right orientation equally often across participants.)

The data indicated a benefit in the processing of studied objects as opposed to nonstudied objects, although the magnitude of the priming effects in this experiment was considerably smaller than the priming obtained in Experiments 1 and 2. The smaller priming effects are likely due to the ease of the left-right judgment task as opposed to the other tasks, reflected in the shorter nonstudied latencies on this task than on other tasks. Priming in the studied conditions was $35 \mathrm{msec}$ in the left-left conditions, $17 \mathrm{msec}$ in the right-right conditions, $39 \mathrm{msec}$ in the right-left conditions, and $17 \mathrm{msec}$ in the left-right conditions. Accuracy data were at ceiling and will not be discussed further.

The main effect of study status was reliable $\left[F_{1}(1,39)=\right.$ $\left.10.58, M S_{\mathrm{e}}=5,530 ; F_{2}(1,79)=9.47, M S_{\mathrm{e}}=8,858\right]$, indicating reliable priming in the studied conditions. To explore the effect of maintaining orientation across study and test for studied items, priming scores were computed for the four study-test conditions and subjected to a separate analysis. There was no reliable effect of study-test compatibility $(F \mathbf{s}<1)$, indicating no adverse effects on priming as a result of a change in orientation between study and test.

Surprisingly, the results indicated no effect of maintaining orientation between study and test despite orienting participants to explicitly encode left-right information at study and at test. One possible explanation for the lack of an effect of orientation in Experiment 4 is the small size of the priming effects observed in this experiment. Thus, the failure to find an effect of changing orientation may be due to the lack of sensitivity of the left-right decision task and to floor effects. This possibility was tested in Experiment 5 by examining the effects of orientation changes on picture fragment naming. Because priming effects on this task tend to be larger in magnitude, it was hypothesized that the test would reveal effects of changes in left-right orientation.

\section{EXPERIMENT 5}

\section{Method}

Participants. Twenty-four Boston College undergraduates participated in this experiment in partial fulfillment of a course requirement.

Design and Materials. The design and materials used in this experiment were identical to those used in Experiment 5, except that fragmented versions of the line drawings were also created for use at test. The fragments were produced by deleting $8 \times 8$ pixel blocks randomly as in Experiment 2.

Procedure. The procedures at study were identical to those in Experiment 4. At test, the participants were instructed to name fragmented versions of the line drawings as quickly and as accurately as possible. The participants pressed a mouse key as soon as they could name the fragment, and then they typed in their responses. Eighty target fragments ( 40 studied and 40 nonstudied) were presented at test; 20 were in the same orientation as at study, and 20 were in a different orientation.

\section{Results and Discussion}

Table 5 presents the latency and accuracy data on the picture fragment task at test.

As can be seen in the table, priming was obtained in all the studied conditions: left-left $(263 \mathrm{msec}, .12)$, rightright ( $324 \mathrm{msec}, .15)$, left-right ( $340 \mathrm{msec}, .11)$, and rightleft (328 msec, .08) study conditions. Repeated measures ANOVAs indicated that these priming effects were reliable both in the latency $\left[F_{1}(1,23)=14.35, M S_{\mathrm{e}}=\right.$ 245,$\left.951 ; F_{2}(1,57)=28.35, M S_{\mathrm{e}}=151,380\right]$ and in the accuracy data $\left[F_{1}(1,23)=45.27, M S_{\mathrm{e}}=.02 ; F_{2}(1,79)=\right.$ $\left.45.56, M S_{\mathrm{e}}=.04\right]$. Separate analysis for the priming scores in the studied conditions indicated no effects of maintaining orientation between study and test in the latency data $(F \mathrm{~S}<1)$ and only a marginally significant effect in the item analysis in the accuracy data $\left[F_{1}(1,23)=\right.$ $\left.1.38, M S_{\mathrm{e}}=.02 ; F_{2}(1,79)=2.91, M S_{\mathrm{e}}=.03 ; p<.10\right]$.

Overall, the data in the picture fragment naming task suggest little or no effects of changing orientation between study and test, which is once again a surprising result, given the sensitivity of the picture fragment naming task to size changes in Experiment 2. One possible explanation for the discrepancy between the two experiments is perhaps to be found in the specific demands of picture fragment naming. Size alteration directly affects

Table 5

Accuracy (PC, Proportion Correct) and Latency (RT, in Milliseconds) in the Picture Fragment Naming Task at Test in Experiment 5

\begin{tabular}{lccccc}
\hline & \multicolumn{5}{c}{ Test Orientation } \\
\cline { 2 - 6 } \multicolumn{1}{c}{ Study } & \multicolumn{2}{c}{ Left } & & \multicolumn{3}{c}{ Right } \\
\cline { 2 - 4 } \cline { 5 - 6 } Orientation & RT & PC & & RT & PC \\
\hline Left & 2,369 & .78 & & 2,344 & .75 \\
Right & 2,304 & .74 & & 2,360 & .79 \\
Nonstudied & 2,632 & .66 & & 2,684 & .64 \\
\hline
\end{tabular}


the ability to complete picture fragments because it affects the spacing between contours, whereas changes in left-right orientation do not affect picture fragment naming, because the change does not affect the overall contour of the fragment. Support for this argument may be found in the data of Experiments 2 and 5: large fragments were easier to name than small fragments in Experiment $2\left[F_{1}(1,63)=23.15, M S_{\mathrm{e}}=177,630 ; F_{2}(1,37)=\right.$ 9.96, $M S_{\mathrm{e}}=242,167$, for latency; $F_{1}(1,5)=49.97$, $M S_{\mathrm{e}}=.03 ; F_{2}(1,47)=23.35, M S_{\mathrm{e}}=.04$, for accuracy], but there was no difference in overall fragment naming performance for left-facing and right-facing fragments $(F \mathrm{~s}<1)$ for latency and accuracy data). Thus, the differential effects of size and orientation on picture fragment naming appear to reflect the differential sensitivity of picture fragment naming to size and left-right orientation changes.

A second possible explanation for the lack of orientation effects on picture fragment naming is that the task of extracting orientation at study was not sufficiently demanding for participants to encode orientation distinctly. This is certainly likely, and it cannot be ruled out by the present experiments. However, it appears that even when the task at study involves the difficult extraction of shape, picture fragment naming is not affected by alterations of left-right orientation. In this study, participants were first presented with intact or fragmented line drawings of objects along with the objects' names, and they were asked to rate the difficulty with which they could identify the fragmented or intact objects (Srinivas \& Roediger, 1996). At test, studied objects were presented with nonstudied objects in the same orientation as at study or in a different orientation. Although the data revealed robust priming effects, no effects of changes in left-right orientation occurred for fragments studied either as intact pictures or as fragments. Clearly, these data suggest that the lack of left-right orientation effects observed in this experiment are not merely due to the relative ease with which the study task was performed.

In the next two experiments, I explored the effects of changes in left-right orientation on recognition memory. Experiment 6 was analogous to the old-new recognition memory test used in Experiment 3 and the test used by L. A. Cooper et al. (1992) with novel objects. In this test, participants were asked to disregard orientation changes when judging whether an object had been studied earlier. Experiment 7 examined memory for left-right orientation of studied objects and was designed to replicate Biederman and E. E. Cooper's (1991a) findings for recognition memory with familiar objects.

\section{EXPERIMENT 6}

\footnotetext{
Method

Participants. Twenty-four Boston College undergraduates participated in this experiment in partial fulfillment of a course requirement.

Design and Materials. The design and materials used in this experiment were identical to those in Experiments 4 and 5.
}

Procedure. The procedures at study were identical to those used in Experiment 5; the participants wete asked to perform the left-right decision task at study. At test, the participants were instructed to indicate whether the object presented on the screen had been studied. They were asked to ignore changes in orientation when making their judgments. They pressed the left mouse key for studied objects and the right mouse key for nonstudied objects. Eighty target items ( 40 studied and 40 nonstudied) were presented at test; 20 were in the same orientation as at study, and 20 were in a different orientation.

\section{Results and Discussion}

Surprisingly, the results on the recognition memory test revealed no effects of changing orientation between study and test; the performance on left-left and rightright conditions was not reliably different from the performance on the right-left and left-right conditions in either the accuracy or the latency data $(F \mathrm{~s}<1$; see Table 6). Thus, the results of this experiment fail to replicate the findings reported by L. A. Cooper et al. (1992). Note however, that L. A. Cooper et al. used novel objects in their experiments to demonstrate the sensitivity of recognition to left-right orientation, whereas the present experiment was conducted with familiar objects.

In fact, recent results suggest that it is difficult to obtain left-right orientation effects in recognition memory for familiar objects when participants are instructed to disregard orientation in making their judgments. For instance, Rajaram (1996) obtained an effect of altering size, but no effect of altering left-right orientation on overall recognition accuracy for familiar objects. Further, the only other experiment that yielded an effect of left-right orientation with familiar objects was explicitly designed to test memory for orientation (Biederman \& E. E. Cooper, 1991a). Thus, in Biederman and E. E. Cooper's (1991a) study, participants were instructed to discriminate studied familiar objects presented in the same orientation from studied objects presented in a different orientation. Because the results of their study revealed that participants could perform this discrimination reliably, Biederman and E. E. Cooper (1991a) argued that reflection information was included in the representations mediating explicit memory, even for familiar objects. Experiment 7 was therefore designed to test whether left-right orientation information is included in the representations mediating explicit memory, although this information is sometimes disregarded in recognition decisions. The instructions, procedure, and

Table 6

Accuracy (PC, Proportion Correct) and Latency (RT, in Milliseconds) in the Recognition Memory Test in Experiment 6

\begin{tabular}{lccccc}
\hline & \multicolumn{4}{c}{ Test Orientation } \\
\cline { 2 - 4 } \multicolumn{1}{c}{ Study } & \multicolumn{2}{c}{ Left } & & \multicolumn{2}{c}{ Right } \\
\cline { 2 - 6 } Orientation & RT & PC & RT & PC \\
\hline Left & 1,008 & .75 & 1,057 & .70 \\
Right & 1,020 & .77 & 1,062 & .68 \\
Nonstudied & 1,053 & .84 & 1,019 & .88 \\
\hline
\end{tabular}


analyses used in this experiment were similar to those used by Biederman and E. E. Cooper (1991a). The participants received the same list at study as in Experiment 6, but at test, they were only presented with studied objects. The instructions were changed in this experiment so that the participants were asked to respond "studied" only if the object had been presented in the same orientation at study. If the participants had failed to encode orientation at study, they would be expected to perform at chance (.50) on this test.

\section{EXPERIMENT 7}

\section{Method}

Participants. Twenty-four Boston College undergraduates participated in this experiment in partial fulfillment of a course requirement.

Design and Materials. The design and materials used in this experiment were similar to those used in Experiment 6, except that the participants were presented with 80 items in one of two orientations at study (left or right) and with the same 80 items at test. Half the items at test were presented in the same orientation, and half in a different orientation.

Procedure. The procedures at study were similar to those used in Experiment 6, except that the participants studied a longer list of items. At test, the participants were instructed to indicate whether the object presented on the screen had been studied before in the exact same orientation. Participants pressed the green (for "same") or red (for "different") mouse key as soon as they could make their judgments.

\section{Results and Discussion}

The results on this modified recognition memory test clearly revealed that participants had encoded studied orientation: accuracy in the left-left $(.80)$ and right-right (.73) conditions was different from chance (where chance was .50). Moreover, this did not seem to be a result of a response bias to respond "same," because correct rejections in the left-right (.59) and right-left (.55) conditions were also better than chance performance. Analyses confirmed these observations both with the combined hits $\left[t_{1}(23)=9.81, t_{2}(79)=13.48\right]$ and with correct rejections $\left[t_{1}(23)=2.38, t_{2}(79)=2.54\right]$. The mean latency for correct responses was $1,708 \mathrm{msec}$.

The results of this experiment replicate Biederman and E. E. Cooper's (1991a) findings regarding memory for left-right orientation for familiar objects. Specifically, participants could use left-right orientation information effectively to discriminate between objects studied in the same orientation and those changed in orientation. Yet, when participants were instructed to disregard orientation information on a recognition memory test, they were clearly able to do so successfully.

\section{GENERAL DISCUSSION}

The goal of the present experiments was to examine whether size and left-right orientation effects could be obtained on perceptual implicit memory tests when the tasks performed at encoding and at retrieval required the processing of this information. Overall, the data sug- gested size effects on perceptual implicit tests that were expected to show such effects because of their task demands. Thus, changes in size between study and test had an adverse effect on a size judgment task (Experiment 1) and on a picture fragment naming task (Experiment 2) because participants had encoded size information at study and both tasks utilized size information. Not surprisingly, recognition memory also revealed an adverse effect of a size change, thus replicating previous results (Biederman \& E. E. Cooper, 1991a; L. A. Cooper et al., 1992; Jolicoeur, 1987; Milliken \& Jolicoeur, 1992).

However, changes in left-right orientation had no effect on perceptual implicit tests, even under conditions that encouraged the processing of orientation information both at study and at test. Thus, little or no adverse effects of changing orientation occurred when participants judged the left-right orientation of an object at study and performed either the same left-right judgment task at test (Experiment 4), or a picture fragment naming task at test (Experiment 5). These results suggest that the sensitivity to processing demands observed with size effects do not generalize to left-right orientation. Further, the alteration of left-right orientation did not affect oldnew discriminations when participants were instructed to ignore orientation changes (Experiment 6). Nevertheless, participants could reliably discriminate between items presented in the same orientation versus those changed in orientation (Experiment 7), suggesting that orientation information is included in the representations that mediate explicit memory. The theoretical implications of these findings are discussed below.

As discussed in the introduction, according to the systems account, the lack of size and orientation effects on perceptual implicit memory tests is due to the fact that these tests tap shape-based representations of objects in the IT that are insensitive to changes in size, left-right orientation, and location (Biederman \& E. E. Cooper, 1992; L. A. Cooper et al., 1992; Schacter, 1992). In contrast, recognition memory is assumed to be affected by changes on these dimensions because these are distinctive spatial attributes of objects that are utilized in episodic memory. Further, some theorists have argued that because size and orientation are spatial or metric attributes, their influence on recognition memory indicates that episodic representations include shape-based information computed by the IT along with spatial information computed by the "where" system (Biederman \& E. E. Cooper, 1992).

However, dissociations between explicit and implicit memory tests also occur because of differences in their processing demands, as predicted by the transferappropriate processing framework (see, e.g., Roediger et al., 1989). One difference in the task demands of typical explicit and implicit tests is that most explicit tests rely heavily on semantic or conceptual processing of items, whereas most implicit tests (such as naming or judgments regarding the three-dimensionality of objects) rely on perceptual processing of items. Of course, this conceptualperceptual difference cannot explain the findings obtained here and in other prior research with regard to the effects 
of changes in size or left-right orientation. As in prior research, the present experiments indicated perceptual effects of size (and to some extent left-right orientation) on a conceptual recognition memory test.

Nevertheless, the framework is useful in exploring the extent to which representations are flexibly encoded in the perceptual representation systems or in the episodic systems (Roediger \& Srinivas, 1993). Specifically, the issue is whether size and left-right orientation information is optionally included in the perceptual representation systems when the perceptual implicit test requires the processing of such information, or when the processing of size and orientation information is encouraged at study. The results provide evidence that on the one hand, size information can be optionally included in the perceptual representations mediating priming. On the other hand, left-right orientation did not appear to be included as easily, despite the fact that orientation information was processed at encoding and at retrieval.

These data suggest that the transfer-appropriate processing principle must be construed as operating within the constraints of different memory systems (see Roediger \& Srinivas, 1993, and Schacter, 1992, for similar arguments). Thus, for instance, if one assumes that the functional property of the perceptual representation systems is to specify the identity of objects across changes in size, left-right orientation or location (or, in the case of words, across changes in type font), then such attributes would be typically excluded or ignored by tasks that tap this system (such as naming and possible/ impossible decisions in previous work). However, as suggested by the findings from the present experiments, some attributes of objects such as size may be optionally included within these systems when the processing of such attributes is encouraged at encoding and/or at retrieval (size judgment task in Experiment 1, and picture fragment naming task in Experiment 2). A similar finding has been reported by Graf and Ryan (1990) with regard to the effects of typography in word identification (a perceptual implicit test). In their experiments, typography manipulations had no effect on priming under elaborative encoding conditions, but did have an effect under conditions that encouraged the processing of the physical features of words at encoding. Together, the data from the present experiments and from prior research (Graf \& Ryan, 1990; Snodgrass \& Hirshman, 1994) emphasize that there is flexibility in the encoding and retrieval operations of a particular perceptual representation system for certain attributes of objects (e.g., size or typography), but not for others (e.g., left-right orientation).

This conceptualization of the perceptual representation systems raises a difficult question for future research. How does one successfully predict the attributes that can be optionally utilized by the perceptual representation systems (e.g., size or type font) versus those attributes that are not utilized even when their processing is encouraged at study and at test (e.g., left-right orientation in the left-right task)? Unfortunately, there is no method that can be used a priori to determine this issue.
At this point, the only speculative explanation of the difference between size and left-right orientation is that size information is sometimes useful in discriminating between different objects (e.g., a volley ball from a golf ball), and may therefore be optionally represented in the system for objects (Roediger \& Srinivas, 1993). In contrast, left-right information is typically never useful in discriminating between different natural objects and hence may not be represented even optionally within this system. If this argument is correct, it would imply that size information should be excluded in the perceptual system for words even if encoding and retrieval of this information is promoted at study and test. In contrast, left-right information would be critical in the perceptual system for words because left-right orientation information can discriminate between certain letters of the alphabet (e.g., "d" vs. "b").

There is no conclusive evidence to support this hypothesis within the domain of verbal perceptual priming tests, either with size or left-right orientation. Indirect evidence regarding the representation of orientation information comes from the work of Kolers and others (e.g., Kolers, 1976; Kolers \& Perkins, 1975), who documented substantial specificity in re-reading transformed text that had been presented earlier. However, the issue of whether these results are due to representations of specific items in a particular orientation, or simply due to the acquisition of a general pattern-analyzing skill is still unresolved (see Tardiff \& Craik, 1989). Similarly, research by Paivio (1975) with a task similar to the size judgment task used in these experiments provides clues regarding the importance of size information for object representations as opposed to verbal representations. Paivio obtained a Stroop-like interference on a size comparison task (where participants decided if a zebra or a lamp was larger when the zebra was presented smaller than the lamp visually) only with pictorial stimuli. When participants were presented with the names of the pictures in a large or small size on the same size comparison task (the word zebra was presented smaller than the word lamp), no interference effects were obtained from the physical size of the words on the screen. In other words, physical size information seems more intimately tied to object representations than to verbal representations. However, the evidence is only suggestive at this point, and the issue of whether size or left-right orientation information is represented for verbal stimuli needs to be tested directly in a standard priming paradigm.

One other somewhat puzzling aspect of the present findings is the differential effects of size and left-right orientation on recognition memory. Note that although the participants were asked to ignore changes in size (Experiment 3) and changes in orientation (Experiment 6) in the two recognition memory experiments, they seemed to be able to successfully ignore encoded orientation (Experiments 6), but not encoded size (Experiment 3). Given that familiar objects were used in both these experiments, it is surprising that encoded size had any effect at all on recognition memory, because the partici- 
pants could have responded simply on the basis of a conceptual code. There is no obvious explanation for this finding; however, it should be pointed out that size and left-right orientation do carry different sorts of information for episodic judgments. For instance, Milliken and Jolicoeur (1992) have pointed out that recognition memory is affected by the distal, and not proximal, size of objects. A change in the distal size of an object typically implies that it is a different object (e.g., if a bird seen yesterday is changed in size today, it usually implies that it is a different bird). Therefore, size may be more important for recollective judgments about objects. In contrast, a change in left-right orientation information does not imply a different object, although the encoding of such spatial information may be important for certain purposes such as navigation (e.g., imagine trying to find a car you parked 20 min ago in a large parking lot; shape information alone would be woefully inadequate). Therefore, left-right information may sometimes be ignored at retrieval when shape information alone is adequate for the recollection. This functional explanation provides a plausible account for the differential weighting of size and left--right orientation information in recognition memory, but it is admittedly speculative and needs to be tested in future research.

\section{REFERENCES}

Biederman, I., \& CoOPER, E. E. (1991a). Evidence for complete translational and reflectional invariance in visual object priming. Perception, 20, 585-593.

Biederman, I., \& CoOPer, E. E. (1991b). Priming contour-deleted images: Evidence for intermediate representations in visual object recognition. Cognitive Psychology, 23, 393-419.

BIEDERMAN, I., \& COOPER, E. E. (1992). Scale invariance in visual object priming. Journal of Experimental Psychology: Human Perception \& Performance, 18, 121-133.

BLAXTON, T. (1989). Investigating dissociations among memory measures: Support for a transfer appropriate processing account of memory. Journal of Experimental Psychology: Learning, Memory, \& Cognition, 15, 657-668

Cooper, L. A., Schacter, D. L., Ballesteros, S., \& Moore, C. (1992). Priming and recognition of three-dimensional objects: Effects of size and reflection. Journal of Experimental Psychology: Learning, Memory, \& Cognition, 18, 43-57.

DavidofF, J. B., \& OstergaARD, A. L. (1988). The role of colour in categorical judgements. Quarterly Journal of Experimental Psychology, 40A, 533-544.

GRAF, P., \& RYAN, L. (1990). Transfer-appropriate processing for implicit and explicit memory. Journal of Experimental Psychology: Learning, Memory, \& Cognition, 16, 978-992.

HamanN, S. B. (1990). Level-of-processing effects in conceptually driven implicit tasks. Journal of Experimental Psychology: Learning, Memory, \& Cognition, 16, 970-977.

JACOBY, L. L. (1983). Remembering the data: Analyzing interactive processes in reading. Journal of Verbal Learning \& Verbal Behavior, 22, 485-508.

JoLICOEUR, P. (1987). A size-congruency effect in memory for visual shape. Memory \& Cognition, 15, 531-543.

KOLERS, P. A. (1976). Reading a year later. Journal of Experimental Psychology: Human Learning \& Memory, 2, 554-556.

Kolers, P. A., \& Perkins, D. N. (1975). Spatial and ordinal components of form perception and literacy. Cognitive Psychology, 7, 228-267.

Kolers, P. A., \& Roediger, H. L., III (1984). Procedures of mind. Journal of Verbal Learning \& Verbal Behavior, 23, 425-449.

Milliken, B., \& Jolicoeur, P. (1992). Size effects in visual recogni- tion memory are determined by perceived size. Memory \& Cognition, 20, 83-95.

Miyashita, Y. (1993). Inferior temporal cortex: Where visual perception meets memory. Annual Review of Neuroscience, 16, 245-263.

Morris, C. D., Bransford, J. D., \& FranKs, J. J. (1977). Levels of processing versus transfer appropriate processing. Journal of Verbal Learning \& Verbal Behavior, 16, 519-533.

Paivio, A. (1975). Perceptual comparisons through the mind's eye. Memory \& Cognition, 3, 635-647.

PlaUt, D. C., \& FARAH, M. J. (1990). Visual object representation: Interpreting neurophysiological data within a computational framework. Journal of Cognitive Neuroscience, 2, 320-343.

Rajaram, S. (1996). Perceptual effects on remembering: Recollective processes in picture recognition memory. Journal of Experimental Psychology: Learning, Memory, \& Cognition, 22, 365-377.

RAPPOLD, V. A., \& HASHTROUDI, S. (1991). Does organization improve priming? Journal of Experimental Psychology: Learning, Memory, \& Cognition, 17, 103-114.

ROEDIGER, H. L., III (1990). Implicit memory: Retention without remembering. American Psychologist, 45, 1043-1056.

ROEDIGER, H. L., III, \& MCDERMOTT, K. B. (1993). Implicit memory in normal human participants. In F. Boller \& J. Grafman (Eds.), Handbook of neuropsychology (Vol. 8, pp. 63-131). Amsterdam: Elsevier.

ROEDIGER, H. L., III, \& SRINIVAS, K. (1993). Specificity of operations in perceptual priming. In P. Graf \& M. E. J. Masson (Eds.), Implicit memory: New directions in cognition, development, and neuropsychology (pp. 17-48). Hillsdale, NJ: Erlbaum.

Roediger, H. L., III, Weldon, M. S., \& Challis, B. H. (1989). Explaining dissociations between explicit and implicit measures of retention: A processing account. In H. L. Roediger III \& F. I. M. Craik (Eds.), Varieties of memory and consciousness: Essays in honour of Endel Tulving (pp. 3-41). Hillsdale, NJ: Erlbaum.

SCHACTER, D. L. (1992). Understanding implicit memory: A cognitive neuroscience approach. American Psychologist, 47, 559-569.

Schacter, D. L., CoOper, L. A., \& Delaney, S. M. (1990). Implicit memory for unfamiliar objects depends on access to structural descriptions. Journal of Experimental Psychology: General, 119, 5-24.

Snodgrass, J. G., \& Feenan, K. (1990). Priming effects in picture fragment completion: Support for the perceptual closure hypothesis. Journal of Experimental Psychology: General, 119, 276-298.

SnOdGRass, J. G., \& Hirshman, E. (1994). Dissociations among implicit and explicit memory tasks: The role of stimulus similarity. Journal of Experimental Psychology: Learning, Memory, \& Cognition, 20, 150-161.

SNOdgrass, J. G., \& VANDERWART, M. (1980). A standardized set of 260 pictures: Norms for name agreement, image agreement, familiarity, and visual complexity. Journal of Experimental Psychology: Human Learning \& Memory, 6, 174-215.

SQUIRE, L. R. (1992). Memory and the hippocampus: A synthesis from findings with rats, monkeys, and humans. Psychological Review, 99, 195-231.

SRINIVAs, K. (1993). Perceptual specificity in nonverbal priming. Journal of Experimental Psychology: Learning, Memory, \& Cognition, 19, 582-602.

SRINIVAS, K., \& RoEdiger, H. L., III (1990). Testing the nature of two implicit tests: Dissociations between conceptually driven and datadriven processes. Journal of Memory \& Language, 29, 389-412.

SRINIVAS, K., \& ROEDIGER, H. L., III (1996). Specificity effects in picture fragment completion. Manuscript in preparation.

TARDIF, T., \& CRAIK, F. I. M. (1989). Reading a week later: Perceptual and conceptual factors. Journal of Memory \& Language, 28, 107-125.

Tulving, E., \& SCHACTER, D. L. (1990). Priming and human memory systems. Science, 247, 301-306.

UNGERLEIDER, L. G., \& MishKIN, M. (1982). Two cortical systems. In D. J. Ingle, M. A. Goodale, R. J. W. Mansfield (Eds.), Analysis of visual behavior (pp. 549-586). Cambridge, MA: MIT Press.

Weldon, M. S., \& Roediger, H. L., III (1987). Altering retrieval demands reverses the picture superiority effect. Memory \& Cognition, $15,269-280$.

(Manuscript received May 8, 1995; revision accepted for publication August 15, 1995.) 